PROCEEDINGS OF THE

AMERICAN MATHEMATICAL SOCIETY

Volume 111, Number 4, April 1991

\title{
A TRANSITIVE MAP ON $[0,1]$ WHOSE INVERSE LIMIT IS THE PSEUDOARC
}

\author{
PIOTR MINC AND W.R.R. TRANSUE
}

(Communicated by James E. West)

\begin{abstract}
We construct a transitive map on $[0,1]$ so that the inverse limit of copies of $[0,1]$ with $f$ as the bonding map is a pseudoarc.
\end{abstract}

M. Barge and J. Martin [4] have extensively investigated relations between inverse limits of maps on the interval and attractors for plane homeomorphisms such as the Hénon maps [8]. They have shown (see [2]) in addition that many of the maps on $[0,1]$ with interesting dynamics give rise to inverse limits which are indecomposable (i.e, are not the union of two proper subcontinua) or at least which contain indecomposable subcontinua. The suspicion is that in fact many of the inverse limits are likely to be hereditarily indecomposable (i.e., all subcontinua are indecomposable). Such objects have been investigated extensively and have been shown by Bing [6] to be homeomorphic to the continuum, first described by Knaster [10] and later investigated by Bing [5] and Moise [11], known as the pseudoarc.

G. Henderson constructed in 1964 [7] a map on [0,1] whose inverse limit is the pseudoarc, but his function, from a dynamic point of view, is less interesting since all orbits save one are attracted to the same fixed point. These considerations led Barge to raise, at the 1988 Spring Topology Conference in Gainesville, Florida, the question "is there a transitive map on $[0,1]$ (i.e., one with an $x$ such that $x, f(x), f(f(x)), \ldots$ is dense in $[0,1])$ whose inverse limit is the pseudoarc?" (This question also appears in [1].) We answer this question in the affirmative.

In [9], J. Kennedy had constructed a transitive homeomorphism on the pseudoarc. Note that each map of $[0,1]$ onto $[0,1]$ induces two homeomorphisms on the inverse limit $[0,1] \stackrel{f}{\longleftarrow}[0,1] \stackrel{f}{\longleftarrow}[0,1] \stackrel{f}{\longleftarrow} \ldots$, namely the right and the left shifts. It should be observed that, if $f$ is transitive, then both of these

Received by the editors May 31, 1989 and, in revised form, March 8, 1990.

1980 Mathematics Subject Classification (1985 Revision). Primary 54H20; Secondary 58F13, 54F20.

Key words and phrases. Inverse limits, transitive map, pseudoarc. 
maps are transitive. Thus our function provides other examples of transitive homeomorphisms on the pseudoarc.

Definition. Let $f:[0,1] \rightarrow[0,1]$ be a continuous function. Let $a$ and $b$ be two points of the interval $[0,1]$, and let $\delta$ be a positive number. We will say that $f$ is $\delta$-crooked between $a$ and $b$ if, for every two points $c, d \in[0,1]$ such that $f(c)=a$ and $f(d)=b$, there is a point $c^{\prime}$ between $c$ and $d$ and there is a point $d^{\prime}$ between $c^{\prime}$ and $d$ such that $\left|b-f\left(c^{\prime}\right)\right| \leq \delta$ and $\left|a-f\left(d^{\prime}\right)\right| \leq \delta$. We will say that $f$ is $\delta$-crooked if it is $\delta$-crooked between every pair of points.

The first three of the following propositions are apparent:

Proposition 1. If $\alpha \geq \delta$, then every $\delta$-crooked map is also $\alpha$-crooked.

Proposition 2. Let $f$ and $F$ be two maps of $[0,1]$ into itself so that $\mid f(t)-$ $F(t) \mid<\varepsilon$ for each $t \in[0,1]$. If $f$ is $\delta$-crooked, then $F$ is $(\delta+2 \varepsilon)$-crooked.

Proposition 3. Let $f_{1}, f_{2}, f_{3}, \ldots$ be a sequence of $\delta$-crooked maps of the interval $[0,1]$ into itself. If the sequence converges uniformly, then the limit is also $\delta$-crooked.

Proposition 4. Let $f:[0,1] \rightarrow[0,1]$ be a continuous map with the property that, for every positive number $\delta$, there is a positive integer $n$ such that $f^{n}$ is $\delta$-crooked. Then the inverse limit of copies of $[0,1]$ with $f$ as the bonding map is a pseudoarc.

Proof. Let $X$ denote the inverse limit of copies of $[0,1]$ with $f$ as the bonding map. Let $p_{i}$ denote the projection of $X$ into the $i$ th interval in the inverse sequence. By [6], it is enough to prove that $X$ is hereditarily indecomposable. Suppose on the contrary that $X$ contains two subcontinua $A$ and $B$ such that the sets $A-B, B-A$, and $A \cap B$ are not empty. There is an integer $k$ such that the sets $p_{k}(A)-p_{k}(B)$ and $p_{k}(B)-p_{k}(A)$ are not empty. The sets $p_{k}(A)$ and $p_{k}(B)$ are intervals; denote them by $\left[a, a^{\prime}\right]$ and $\left[b^{\prime}, b\right]$. Without loss of generality, we can assume that $a<b^{\prime} \leq a^{\prime}<b$. Take a positive number $\delta<\min \left(b^{\prime}-a, b-a^{\prime}\right)$. There is an integer $n$ such that $f^{n}$ is $\delta$-crooked. This leads to a contradiction, because $f^{n}\left(p_{k+n}(A)\right)=\left[a, a^{\prime}\right], f^{n}\left(p_{k+n}(B)\right)=\left[b^{\prime}, b\right]$, and $p_{k+n}(A)$ and $p_{k+n}(B)$ are two intervals with nonempty intersection.

For a positive number $r$ and $A \subset[0,1]$, let $\mathscr{B}(A, r)=\{x \in[0,1] \mid \exists y \in A$ with $|x-y| \leq r\}$, and let $\operatorname{diam}(A)$ denote the diameter of $A$.

Proposition 5. Let $\varepsilon<1$ and $\gamma<\varepsilon / 4$ be two positive numbers. Then there is a piecewise linear and continuous map $g:[0,1] \rightarrow[0,1]$ such that

(i) $|t-g(t)|<\varepsilon / 2+\gamma$ for each $t \in[0,1]$,

(ii) for every $a$ and $b$ such that $|a-b|<\varepsilon, g$ is $\gamma$-crooked between $a$ and $b$, 
and for each subinterval $A$ of $[0,1]$ we have

(iii) $\operatorname{diam}(g(A)) \geq \operatorname{diam}(A)$,

and if, additionally, $\operatorname{diam}(A) \geq \gamma$, then

(iv) $\operatorname{diam}(g(A))>\varepsilon / 2$,

(v) $A \subset g(A)$, and

(vi) $g(B) \subset \mathscr{B}(g(A), r+\gamma)$ for each real number $r$ and each set $B \subset$ $\mathscr{B}(A, r)$.

Proof. Let $q$ be an integer such that $q \gamma \geq 4$. Let $\mu=1 / q$ and let $p$ be the integer so that $\mu(p-2)<\varepsilon / 2 \leq \mu(p-1)$. For each $i=0, \ldots, q-1$, let $u_{i}=(i-\min (i, p)) \mu$ and $v_{i}=(i+\min (q-i, p)) \mu$. On each interval of the form $[i \mu,(i+1) \mu]$ the function $g$ is defined in the following way: $g(i \mu)=u_{i}$; then we move up to $v_{i}$ in crooked fashion, joining linearly the values $u_{i}, u_{i}+\mu$, $u_{i}+2 \mu, u_{i}+\mu, u_{i}+2 \mu, u_{i}+3 \mu, u_{i}+2 \mu, u_{i}+\mu, u_{i}+2 \mu, u_{i}+3 \mu, u_{i}+2 \mu$, $u_{i}+3 \mu, u_{i}+4 \mu, \ldots$, (see [5]), and then move crookedly down to $u_{i+1}$, a goal which is achieved at $(i+1) \mu$. It may be verified that a map $g$ so defined has the required properties.

Definition. A continuous and piecewise linear function $f:[0,1] \rightarrow[0,1]$, will be called admissible if $\left|f^{\prime}(t)\right| \geq 4$ for every $t$ such that $f^{\prime}(t)$ exists and for every $0 \leq a<b \leq 1$ there is a positive integer $m$ such that $f^{m}([a, b])=$ $[0,1]$.

The proof of the next proposition is easy, and it will therefore be omitted.

Proposition 6. Let $f:[0,1] \rightarrow[0,1]$ be a continuous map with the property that, for every $0 \leq a<b \leq 1$, there is a positive integer $m$ such that $f^{m}([a, b])=[0,1]$. Then for every positive number $\varepsilon$ there is a positive integer $n$ such that $f^{n}([a, b])=[0,1]$ for every $0 \leq a<b \leq 1$ such that $b-a \geq \varepsilon$.

Lemma. Let $f:[0,1] \rightarrow[0,1]$ be an admissible map. Let $\eta$ and $\delta$ be two positive numbers. Then there is an admissible map $F:[0,1] \rightarrow[0,1]$ and there is a positive integer $n$ such that $F^{n}$ is $\delta$-crooked and $|F(t)-f(t)|<\eta$ for every $t \in[0,1]$. Moreover, if $0 \leq a<b \leq 1$ and $b-a \geq \eta$, then $f([a, b]) \subset F([a, b])$ and $F^{n}([a, b])=[0,1]$.

Proof. Since $f$ is piecewise linear, there is a positive number $\alpha$ such that, if $0 \leq a<b \leq 1$ and $b-a<\alpha$, then between $a$ and $b$ there is a point $c$ such that $f$ is linear on both intervals $[a, c]$ and $[c, b]$. Since $\left|f^{\prime}(t)\right| \geq 4$ for $t \in(a, c) \cup(c, b)$, it follows that

$$
\begin{aligned}
& \operatorname{diam}(f([a, b])) \geq 2(b-a) \text { for every } a \text { and } b \text { such that } \\
& 0 \leq a<b \leq 1 \text { and } b-a<\alpha .
\end{aligned}
$$

There is a number $s$ such that $\left|f^{\prime}(t)\right|<s$ for every $t$ such that $f^{\prime}(t)$ exists. Observe that

$$
\operatorname{diam}(f(C)) \leq s \operatorname{diam}(C) \text { for every } C \subset[0,1]
$$


Set $\varepsilon=\eta / s$. There is a positive integer $n$ such that, if $0 \leq a<b \leq 1$ and $b-a>\varepsilon / 4$, then $f^{n}([a, b])=[0,1]$ (see Proposition 6).

Let $\gamma$ be a positive real number less than $\min \left(\alpha, s^{-n}, \varepsilon / 4, \delta s^{-n} / 5\right)$.

It follows from (1) that

$$
\operatorname{diam}(f(A)) \geq 2 \min (\operatorname{diam}(A), \gamma) \text { for each interval } A \subset[0,1] .
$$

Let $g$ be a map satisfying Proposition 5. Define $F=f g$ (composition). Note that $F$ is a piecewise linear map. It follows from Proposition 5(iii) that $\left|F^{\prime}(t)\right| \geq 4$ for every $t$ such that $F^{\prime}(t)$ exists. Since $\gamma<\eta$, it follows from Proposition 5(v) that, if $0 \leq a<b \leq 1$ and $b-a \geq \eta$, then $[a, b] \subset g([a, b])$ and consequently $f([a, b]) \subset F([a, b])$.

Since $|g(t)-t|<\eta / s$ and $|f g(t)-f(t)| \leq s|g(t)-t|$, we have the result that $|F(t)-f(t)|<\eta$ for each $t \in[0,1]$.

Now, we will prove that

if $C$ is a subinterval of $[0,1]$ such that $\operatorname{diam}(C)>\varepsilon / 4$, then $F^{n-1} f(C)=[0,1]$.

First, observe that, since $f^{n}(C)=[0,1]$ and $\gamma \leq s^{-n}$, it follows from (2) that, for each positive integer $j, \operatorname{diam}\left(f^{j}(C)\right) \geq \gamma$. Using Proposition $5(\mathrm{v})$ inductively, we get the result that $f^{j}(C) \subset F^{j-1} f(C)$ for $j=1, \ldots, n$. The condition (4) follows from the last inclusion.

If $0 \leq a<b \leq 1$ and $b-a \geq \gamma$ then, by Proposition 5(iv), there is a point $c \in(a, b)$ such that $\operatorname{diam}(g([a, c]))>\varepsilon / 4$ and $\operatorname{diam}(g([c, b]))>\varepsilon / 4$. It follows from (4) that

if $0 \leq a<b \leq 1$ and $b-a \geq \gamma$, then there is a point $c \in(a, b)$

such that $F^{n}([a, c])=[0,1]$ and $F^{n}([c, b])=[0,1]$. In particular, $F^{n}([a, b])=[0,1]$.

To prove that $F$ is admissible, it remains to show that if $0 \leq a<b \leq 1$, then some iteration of $F$ maps $[a, b]$ onto $[0,1]$. It follows from (1) and Proposition 5 (iii) that there is a nonnegative integer $m$ such that $\operatorname{diam}\left(F^{m}([a, b])\right) \geq$ $\gamma$. By $(5), F^{m+n}([a, b])=[0,1]$.

To prove that $F^{n}$ is $\delta$-crooked, we need the following claim.

(6) Claim. Let $A$ be a subinterval of $[0,1]$ such that $\operatorname{diam}(A) \geq \gamma$. Let $B$ be a subset of $[0,1]$, and let $r$ be a number such that $B \subset \mathscr{B}(A, r)$. For each positive integer $j, F^{j}(B) \subset \mathscr{B}\left(F^{j}(A), q\right)$, where $q=s^{j}(r+2 \gamma)$.

By (3) and Proposition 5(iii), we have the result that $\operatorname{diam}\left(F^{i}(A)\right) \geq \gamma$ for each positive integer $i$. Set $q_{0}=r$ and $q_{i+1}=s\left(q_{i}+\gamma\right)$ for $i=0,1, \ldots$ Repeatedly using Proposition 5(vi) and (2), one can prove $F^{j}(B) \subset \mathscr{B}\left(F^{j}(A), q_{j}\right)$. Observe that $q_{j}=s^{j} r+\gamma \frac{s^{j+1}-1}{s-1}<s^{j}\left(r+\frac{s}{s-1} \gamma\right)<s^{j}(r+2 \gamma)$, so the claim is true.

Let $a$ and $b$ be two arbitrary points from $[0,1]$. We will prove that $F^{n}$ is $\delta$-crooked between $a$ and $b$. Obviously, we can assume that $a<b$ and 
$b-a \geq 2 \delta$. Let $I$ be a subinterval of $[0,1]$, so that $[a, b] \subset F^{n}(I)$. In order to prove that $F^{n}$ is $\delta$-crooked between $a$ and $b$, it is enough to show that there are two disjoint subintervals $A_{1}$ and $A_{2}$ of $I$ such that $[a+\delta, b-\delta] \subset F^{n}\left(A_{1}\right) \cap F^{n}\left(A_{2}\right)$.

By (5) we can assume that $\operatorname{diam}(I)<\gamma$. Observe that $\operatorname{diam}\left(F^{n}(I)\right) \geq 2 \delta>$ $\gamma$. Let $m$ be the greatest integer such that $\operatorname{diam}\left(F^{m}(I)\right)<\gamma$. Denote $F^{m}(I)$ by $M$. We will consider the two cases: $\operatorname{diam}(g(M))>2 \gamma$ and $\operatorname{diam}(g(M)) \leq 2 \gamma$. Case. $\operatorname{diam}(g(M))>2 \gamma$.

Let $c$ and $d$ be the endpoints of the interval $g(M)$. Assume that $c<d$. Since $\operatorname{diam}(M)<\gamma$, it follows from Proposition 5(i) that $d-c<\varepsilon+3 \gamma$. There are points $c_{1}$ and $d_{1}$ such that $c \leq c_{1}<d_{1} \leq d, 2 \gamma<d_{1}-c_{1}<\varepsilon$, and $[c, d] \subset$ $\mathscr{B}\left(\left[c_{1}, d_{1}\right], 3 \gamma\right)$. Since, by Proposition 5(ii), $g$ is $\gamma$-crooked between $c_{1}$ and $d_{1}$, there are two disjoint subintervals $M_{1}$ and $M_{2}$ of $M$ such that $g\left(M_{1}\right)=$ $\left[c_{1}, d_{1}-\gamma\right]$ and $g\left(M_{2}\right)=\left[c_{1}+\gamma, d_{1}\right]$. There are two disjoint subintervals $A_{1}$ and $A_{2}$ of $I$ such that $F^{m}\left(A_{k}\right)=M_{k}$ for $k=1,2$. Observe that $g(M) \subset$ $\mathscr{B}\left(g\left(M_{k}\right), 4 \gamma\right)$, and it follows from (2) that $F(M) \subset \mathscr{B}\left(F\left(M_{k}\right), 4 s \gamma\right)$ for $k=1,2$. By (3), $\operatorname{diam}\left(F\left(M_{k}\right)\right)>\gamma$. It follows from (6) that $F^{n}(I)=$ $F^{n-m}(M) \subset \mathscr{B}\left(F^{n-m}\left(M_{k}\right), s^{n-m-1}(4 s \gamma+2 \gamma)\right)$. Since $S^{n-m-1}(4 s \gamma+2 \gamma)<$ $5 \gamma s^{n}<\delta,[a+\delta, b-\delta] \subset F^{n-m}\left(M_{1}\right) \cap F^{n-m}\left(M_{2}\right)=F^{n}\left(A_{1}\right) \cap F^{n}\left(A_{2}\right)$.

Case. $\operatorname{diam}(g(M)) \leq 2 \gamma$.

Let $u$ and $v$ be the endpoints of the interval $g F(M)$. Assume that $u<v$. By (2) and the choice of $m$, we have the result that $\gamma \leq \operatorname{diam}(F(M)) \leq 2 \gamma s$. It follows from Proposition 5(i) and (iv) that $\varepsilon / 2<v-u<\varepsilon+2 \gamma(s+1)$. There are points $u_{1}$ and $v_{1}$ such that $u \leq u_{1}<v_{1} \leq v, 2 \gamma<v_{1}-u_{1}<\varepsilon$, and $[u, v] \subset \mathscr{B}\left(\left[u_{1}, v_{1}\right], 2 \gamma(s+1)\right)$. Since, by Proposition 5(ii), $g$ is $\gamma$-crooked between $u_{1}$ and $v_{1}$, there are two disjoint subintervals $N_{1}$ and $N_{2}$ of $F(M)$ such that $g\left(N_{1}\right)=\left[u_{1}, v_{1}-\gamma\right]$ and $g\left(N_{2}\right)=\left[u_{1}+\gamma, v_{1}\right]$. There are two disjoint subintervals $A_{1}$ and $A_{2}$ of $I$ such that $F^{m+1}\left(A_{k}\right)=N_{k}$ for $k=1,2$. Observe that $g F(M) \subset \mathscr{B}\left(g\left(N_{k}\right), 2 \gamma(s+1)+\gamma\right) \subset \mathscr{B}\left(g\left(N_{k}\right), 4 \gamma s\right)$, and it follows from (2) that $F^{2}(M) \subset \mathscr{B}\left(F\left(N_{k}\right), 4 s^{2} \gamma\right)$ for $k=1,2$. By (3), $\operatorname{diam}\left(F\left(N_{k}\right)\right)>\gamma$. It follows from (6) that

$$
F^{n}(I)=F^{n-m}(M) \subset \mathscr{B}\left(F^{n-m-1}\left(N_{k}\right), s^{n-m-2}\left(4 s^{2} \gamma+2 \gamma\right)\right) .
$$

Since $s^{n-m-2}\left(4 s^{2} \gamma+2 \gamma\right)<5 \gamma s^{n}<\delta,[a+\delta, b-\delta] \subset F^{n-m-1}\left(N_{1}\right) \cap F^{n-m-1}\left(N_{2}\right)$ $=F^{n}\left(A_{1}\right) \cap F^{n}\left(A_{2}\right)$, and the proof of the lemma is complete.

Theorem. There is a transitive map $f:[0,1] \rightarrow[0,1]$ such that the inverse limit of copies of $[0,1]$ with $f$ as the bonding map is a pseudoarc.

Proof. We are going to construct a sequence of positive integers $n(1), n(2)$, $n(3), \ldots$ and a sequence $f_{1}, f_{2}, f_{3}, \ldots$ of admissible maps of the interval $[0,1]$ into itself such that, for each positive integer $i$, the following conditions are satisfied:

(i) $\left|f_{i+1}(t)-f_{i}(t)\right|<2^{-i}$ for each $t \in[0,1]$, 
(ii) $f_{i}^{n(k)}$ is $\left(2^{-k}-2^{k-i}\right)$-crooked for each positive integer $k \leq i$, and

(iii) if $0 \leq a<b \leq 1$ and $b-a \geq 2^{-k}$, then $f_{i}^{n(k)}([a, b])=[0,1]$ for each positive integer $k \leq i$.

It is easy to construct $n(1)$ and $f_{1}$. We assume that $n(1), \ldots, n(i-1)$ and $f_{1}, \ldots, f_{i-1}$ have already been constructed, and we will construct $n(i)$ and $f_{i}$.

There is a positive number $\eta<2^{-i}$ such that, if $F:[0,1] \rightarrow[0,1]$ such that $\left|f_{i-1}(t)-F(t)\right|<\eta$ for each $t \in[0,1]$, then $\left|f_{i-1}^{n(k)}(t)-F^{n(k)}(t)\right|<2^{-k-i-1}$ for each $t \in[0,1]$ and for each positive integer $k<i$. Note that $\eta$ is chosen such that $F^{n(k)}$ is $\left(2^{-k}-2^{-k-i}\right)$-crooked (see Proposition 2). Now, use the lemma with $f=f_{i-1}$ and $\delta=2^{-i}-2^{-i-i}$. Define $f_{i}=F$ and $n(i)=n$. It is easy to see that conditions (i) and (ii) of the construction are satisfied. To prove (iii), it is enough to observe that if $b-a \geq 2^{-i}>\eta$, then $f_{i-1}([a, b]) \subset f_{i}([a, b])$.

By (i), the sequence $f_{1}, f_{2}, f_{3}, \ldots$ converges uniformly. Denote the limit by $f$. Apply Propositions 1 and 3 , we get the result that $f^{n(k)}$ is $\left(2^{-k}\right)$-crooked for each positive integer $k$. Applying Propositions 1 and 4, we get the result that the inverse limit of copies of $[0,1]$ with $f$ as the bonding map is a pseudoarc.

Observe that condition (iii) of the construction implies that, if $0 \leq a<b \leq$ 1 and $b-a \geq 2^{-k}$, then $f^{n(k)}([a, b])=[0,1]$. Therefore it follows from Theorem 6 of [3] that $f$ is transitive.

\section{REFERENCES}

1. M. Barge and J. Kennedy, Continuum theory and topological dynamics, Open problems in topology (J. van Mill and G. M. Reed, eds.) North-Holland, Amsterdam (to appear).

2. M. Barge and J. Martin, Chaos, periodicity and snake-like continua, Trans. Amer. Math. Soc. 289 (1985), 355-365.

3. __ Dense orbits on the interval, Michigan Math. J. 34 (1987), 3-11.

4. _ The construction of global attractors, Proc. Amer. Math. Soc. (to appear).

5. R. H. Bing, A homogeneous indecomposable plane continuum, Duke Math. J. 15 (1948), 729-742.

6. __ Snake-like continua, Duke Math. J. 18 (1951), 653-663.

7. G. W. Henderson, The pseudo-arc as an inverse limit with one binding map, Duke Math. J. 31 (1964), 421-425.

8. M. Hénon, A two dimensional mapping with a strange attractor, Comm. Math. Phys. $\mathbf{5 0}$ (1976), 69-77.

9. J. Kennedy, A transitive homeomorphism on the pseudoarc which is semiconjugate to the tent map, preprint.

10. B. Knaster, Un continu dont tout sous-continu est indécomposable, Fund. Math. 3 (1922), 247-286.

11. E. E. Moise, An indecomposable plane continuum which is homeomorphic to each of its nondegenerate subcontinua, Trans. Amer. Math. Soc. 63 (1948), 581-594.

Department of Mathematics, Auburn University, Auburn, Alabama 36849 